\title{
Análisis de la sustentabilidad económica de la producción de papa en la región Lima
}

\author{
Analysis of the economic sustainability of potato \\ production in the Lima region \\ ${ }^{1}$ Sergio Eduardo Contreras Liza ${ }^{a},{ }^{2}$ Alberto Valenzuela Muñoz ${ }^{b}$, \\ ${ }^{3}$ Sady García Bendezú ${ }^{a}$
}

\section{RESUMEN}

Determinar el grado de sustentabilidad económica en las parcelas de producción de papa y proponer alternativas de manejo agronómico en la región Lima. La investigación se realizó en las provincias de Barranca, Huaral y Cañete (región Lima) utilizándose encuestas estructuradas a 127 productores de papa para medir el nivel de sustentabilidad. Se aplicó la metodología multicriterio de Sarandon que considera indicadores y sub-indicadores de sustentabilidad de un agro-ecosistema en la dimensión económica. Las fincas productoras de papa en la región Lima, presentaron un valor ponderado de 1.82 equivalente a una débil sustentabilidad económica, debajo del umbral determinado en 2.0, siendo los puntos críticos del sistema de producción de papa, una precaria autosuficiencia alimentaria y el mayor riesgo económico atribuido a los canales de comercialización y la poca diversificación para la venta. La producción de papa en la región Lima presentó una débil sustentabilidad económica, implicando que ello podría constituirse en una amenaza para la producción sostenible de papa en costa central del Perú.

Palabras clave: indicadores de sustentabilidad, análisis multicriterio, encuestas, costa central.

\begin{abstract}
To determine the degree of economic sustainability in the potato production system and to propose alternatives for agronomic management in the Lima region. The research was conducted in the provinces of Barranca, Huaral and Cañete (Lima region), using structured surveys for 127 potato producers to measure the level of sustainability. The multi-criteria methodology of Sarandon was applied, which considers indicators and sub-indicators of sustainability of an agro-ecosystem in the economic dimension. The potato farms in the Lima region presented a weighted value of 1.82 equivalent to a weak economic sustainability, below the threshold determined in 2.0 , being the critical points of the potato production system, a precarious food self-sufficiency and highest economic risk attributed to deficient marketing channels and low diversification for marketing. Potato production in the Lima region showed weak economic sustainability, implying that this could constitute a threat to sustainable potato production in the central coast of Peru.
\end{abstract}

Key words: sustainability indicators, multicriteria analysis, surveys, central coast.

\footnotetext{
${ }^{1}$ Universidad Nacional José Faustino Sánchez Carrión

${ }^{a}$ Ing. Agrónomo, ${ }^{b}$ Economista
} 


\section{INTRODUCCIÓN}

En la región Lima provincias prevalece el sistema de parcelas agrícolas originadas como consecuencia de 1 as desmembración de las ex Cooperativas Agrarias de Producción (CAPs) establecidas en la Reforma Agraria de la década de 1970, siendo aún hoy el modelo económico predominante de organización campesina. Las parcelas o fincas son las unidades productivas básicas de la agricultura en la región costa y son conducidas por agricultores que utilizan mayormente tecnología agrícola $\mathrm{y}$ hacen por lo general uso de capital financiero. El desempeño de las fincas y la capacidad de gestión de los agricultores dependen de características del entorno y la tecnología empleada. Es necesario por lo tanto, conocer los elementos estructurales y funcionales de los sistemas parcelarios y evaluarlos para explicar su desempeño desde una perspectiva sistémica, lo que servirá para diseñar modelos alternativos o de ajuste para optimizarlos.

Más del $80 \%$ del área dedicada a la papa es cultivada por pequeños agricultores, con una superficie menor a 20 hectáreas; actualmente se estima la superficie agrícola de papa en más de 300 mil hectáreas a nivel nacional (Eguren, 2012), siendo su rendimiento promedio nacional de 13,4 t.ha-1 (MINAGRI, 2015). Se conoce que el cultivo es muy sensible a una serie de estreses ambientales bióticos y abióticos; estos problemas, aunados al hecho que la especie se propaga comercialmente por medios vegetativos hacen que la sanidad del cultivo, sea un problema considerable para la producción de material de siembra de calidad y para la producción comercial (CIP, 2014). De acuerdo a Maldonado et al. (2008) en las década de 1960-2000, la producción de papa en el Perú se incrementó de 1.3 a 3 millones de toneladas anuales. Este volumen de producción ha convertido al país en el mayor productor de papa en América Latina. La importancia que tiene la producción de papa en nuestro país es considerable y por esta razón es necesario explorar los niveles de sustentabilidad ambiental, económica y social del sistema de producción, caracterizando el sistema de producción predial y proponiendo alternativas agroecológicas que permitan mejorar el nivel de vida de los productores de papa.

La agricultura sustentable generalmente se refiere a un modo de producción agrícola que intenta proveer rendimientos sostenidos durante largo tiempo mediante el uso de tecnologías ecológicamente probadas. La agricultura no solo se orienta para obtener altos rendimientos de algún producto, sino más bien para optimizar el sistema entero. Las metodologías denominadas de "investigación de sistemas prediales o de finca" enfatizan la comprensión de los sistemas agrícolas tradicionales como punto de partida, evalúan los antecedentes de la zona,

$\begin{array}{llllllll}r & \text { e } & \text { a } & 1 & \text { i } & z & \text { a } & n \\ \text { Rev. Tayacaja 1 (2): } & 21 & -30,2018 & \text { ISSN:2617-9156 } & \mathbf{2 2}\end{array}$


exámenes en el terreno que incluyen entrevistas a los agricultores respecto a las características del predio y analizan acerca del porqué los agricultores emplean métodos particulares de producción(Altieri, 1997).

Para Masera et al. (1999), el concepto de sustentabilidad se define a partir de cinco atributos generales de los agroecosistemas o sistemas de manejo: (a) productividad; (b) estabilidad, confiabilidad y resiliencia; (c) adaptabilidad; (d) equidad; (e) autodependencia. Sin embargo, no existe un conjunto de indicadores universales que puedan ser utilizados para cualquier situación. Las diferencias existentes en la escala de trabajo (finca, región, etc.), el tipo de fincas, los objetivos deseados, la actividad productiva, las características de los agricultores, hacen imposible su generalización (Sarandon y Flores, 2014).

Sarandon \& Flores (2014) proponen pautas metodológicas para la evaluación de la sustentabilidad de los agroecosistemas y para determinar si el proceso de la transición de sistemas convencionales hacia sistemas diversificados y sustentables está sentado en principios y procesos agroecológicos; de esta manera, los autores describen y analizan una metodología multicriterio para la construcción y uso de indicadores de sustentabilidad. Originalmente, la propuesta desarrollada por Sarandón (2002) consiste en una serie de pasos que conducen como producto final, a la obtención de indicadores adecuados para evaluar los puntos críticos de la sustentabilidad de los agro-ecosistemas. El área seleccionada para este estudio abarcó tres localidades productoras de papa en la región Lima: Barranca, Huaral y Cañete; el agro-ecosistema de Costa se caracteriza por presentar suelos aluviales franco arenosos con baja cantidad de materia orgánica y aptos para el cultivo de papa en temporada de invierno.

Este trabajo de investigación tuvo el objetivo de evaluar la sustentabilidad económica de las fincas productoras de papa y proponer alternativas de gestión para optimizar el sistema de producción de papa bajo las condiciones de la costa central del Perú.

\section{MATERIAL Y MÉTODOS}

Localización: La investigación se realizó en las provincias de Barranca, Huaral y Cañete, principales zonas productoras con el 31, 24 y 15\% respectivamente del área sembrada con papa en Costa central (MINAGRI, 2015). En las zonas de estudio, el clima es desértico y semicálido, con pequeñas oscilaciones de temperatura $\left(8^{\circ} \mathrm{C}\right.$ en promedio al año), y ligeras lloviznas (del orden de $7 \mathrm{~mm}$ al año) que se producen en los meses de invierno y alto porcentaje de humedad relativa (hasta $100 \%$ en invierno). La temperatura máxima media fue de $29.7^{\circ}$ C, correspondiente al mes de marzo, y la temperatura mínima media fue de $13.1^{\circ} \mathrm{C}$ 
en el mes de julio. La humedad relativa media en los meses de primavera fue de $72 \%$. Asimismo, la evaporación total mensual varió dentro de un rango de 222 $\mathrm{mm}$ y $71 \mathrm{~mm}$, durante las estaciones de invierno-primavera.

Tipo de Investigación: El tipo de investigación es no experimental, transversal y de naturaleza descriptiva. La hipótesis de trabajo fue que los sistemas de producción de papa en la región Lima presentan atributos e indicadores con un adecuado nivel de sustentabilidad económica. Un sistema se considera sustentable si mantiene o mejora el capital económico, ya que éste viabiliza el capital natural, mediante una adecuada gestión de los recursos disponibles.

Diseño de la investigación: Se aplicó la metodología de Sarandon (2002) que considera indicadores y sub-indicadores de sustentabilidad de un agro-ecosistema en 3 niveles o dimensiones: económica, ecológica y social. En este caso utilizamos indicadores económicos (IK), adaptados de la metodología citada, con el supuesto de que los aspectos que fortalecen las relaciones económicas en la finca, son favorables a la sustentabilidad; la correcta elección de los indicadores apropiados, depende de la capacidad de comprensión del funcionamiento del sistema (Sarandón 2002). Los indicadores evaluados fueron:
A. Autosuficiencia alimentaria
B. Ingreso neto mensual por grupo

C. Riesgo económico,

Se usó una escala cuantitativa de 0 (muy pobre sustentabilidad) hasta 4 (alto nivel de sustentabilidad). Se consideró el valor de 2 como un nivel aceptable de sustentabilidad (umbral), según Sarandon y Flores (2014).

Ponderaciones IK (Sustentabilidad Económica):

Para estimar las ponderaciones a los indicadores de sustentabilidad, se hicieron consultas a diversos especialistas del sector agrario y dichos valores fueron utilizados para fines de la investigación. Con ello, se utilizaron los siguientes valores:

$$
\begin{aligned}
& \mathrm{IK}=[0,15(\mathrm{~A} 1)+0,10(\mathrm{~A} 2)]+[0,10 \\
& (\mathrm{B} 1)+0,10(\mathrm{~B} 2)]+[0,10(\mathrm{C} 1)+0,10(\mathrm{C} 2) \\
& +0,10(\mathrm{C} 3)+0,20(\mathrm{C} 4)]=
\end{aligned}
$$

Dónde:

A, Autosuficiencia alimentaria, con los siguientes sub-indicadores:

A1: Diversificación de la producción

A2: Superficie de autoconsumo

$\mathrm{B}$, Ingreso neto mensual por grupo, con los siguientes sub-indicadores:

B1: Ingreso neto mensual por familia

B2: Nivel tecnológico del productor

C, Riesgo Económico, con los siguientes sub-indicadores:

C1: Diversificación para la venta

C 2: N úmero de canales de comercialización

C3: Dependencia de insumos externos

C4: Variabilidad en fechas de siembra de papa. 


\section{Instrumentos de medición de los indicadores}

Se utilizó la encuesta a los productores de papa mediante un cuestionario de preguntas dirigidas a obtener información válida de las variables e indicadores de sustentabilidad. El número de entrevistados $(\mathrm{N})$ en las tres provincias productoras de papa en Costa (Barranca, Huaral y Cañete) fue de 96, estimado mediante la expresión basada en Scheaffer, Mendenhall \& Ott (1987):

$\mathrm{N}=\mathrm{Z}^{2}$ p.q $/ \mathrm{e}^{2}=96$.

$\mathrm{Z}^{2}=1,96$ (Probabilidad para una confianza del $95 \%$ en distribución de 2 colas de Z)

$\mathrm{p}=\mathrm{q}=0,5$ (para variables dicotómicas, con varianza igual a p.q)

$\mathrm{e}=$ error de muestreo estimado, considerado para la investigación en $10 \%$.

\section{Tratamiento estadístico de la información}

Los datos que se obtuvieron de las muestras de 127 fincas productoras de papa en la región Lima fueron procesadas mediante el programa estadístico $\mathrm{R}$ ( $\mathrm{R}$ Core Team, 2015). Para el cálculo de los indicadores se procedió según Sarandon (1998), utilizándose una valoración estandarizada para cada sub-indicador de sustentabilidad económica, mediante la siguiente expresión:

Estandarización $=($ Promedio-Valor mínimo)*(Rango/Mediana)

Para ello se hizo uso de una hoja de cálculo Excel y se graficaron los resultados para los indicadores mediante una plantilla ameba o radial.

\section{RESULTADOS}

En la tabla 1, se muestran los valores estandarizados de los indicadores económicos propuestos según la metodología utilizada y finalmente el valor estimado de dicho indicador ponderado (IK) que para el caso de las fincas productoras de papa en la región Lima fue de 1,82 equivalente a una débil sustentabilidad económica. La etapa de estandarización y ponderación, aunque facilita enormemente el análisis, tiene un alto componente de subjetividad (Sarandón, 2002). Sin embargo, esta puede disminuir cuanto mayor sea la información sobre el rol que cada componente tenga sobre la sustentabilidad del sistema en cuestión; el bajo valor hallado de la sustentabilidad económica en el estudio puede mostrar una amenaza futura para las fincas productoras de papa en Costa. 
Tabla 1. Indicadores de sustentabilidad económica de las fincas productoras de papa en la región Lima, según el análisis multicriterio de Sarandon (2002)

\section{Variable Sadindicador VEstándar sponderadón VPonderado DE N Min Max. Medana Rango}

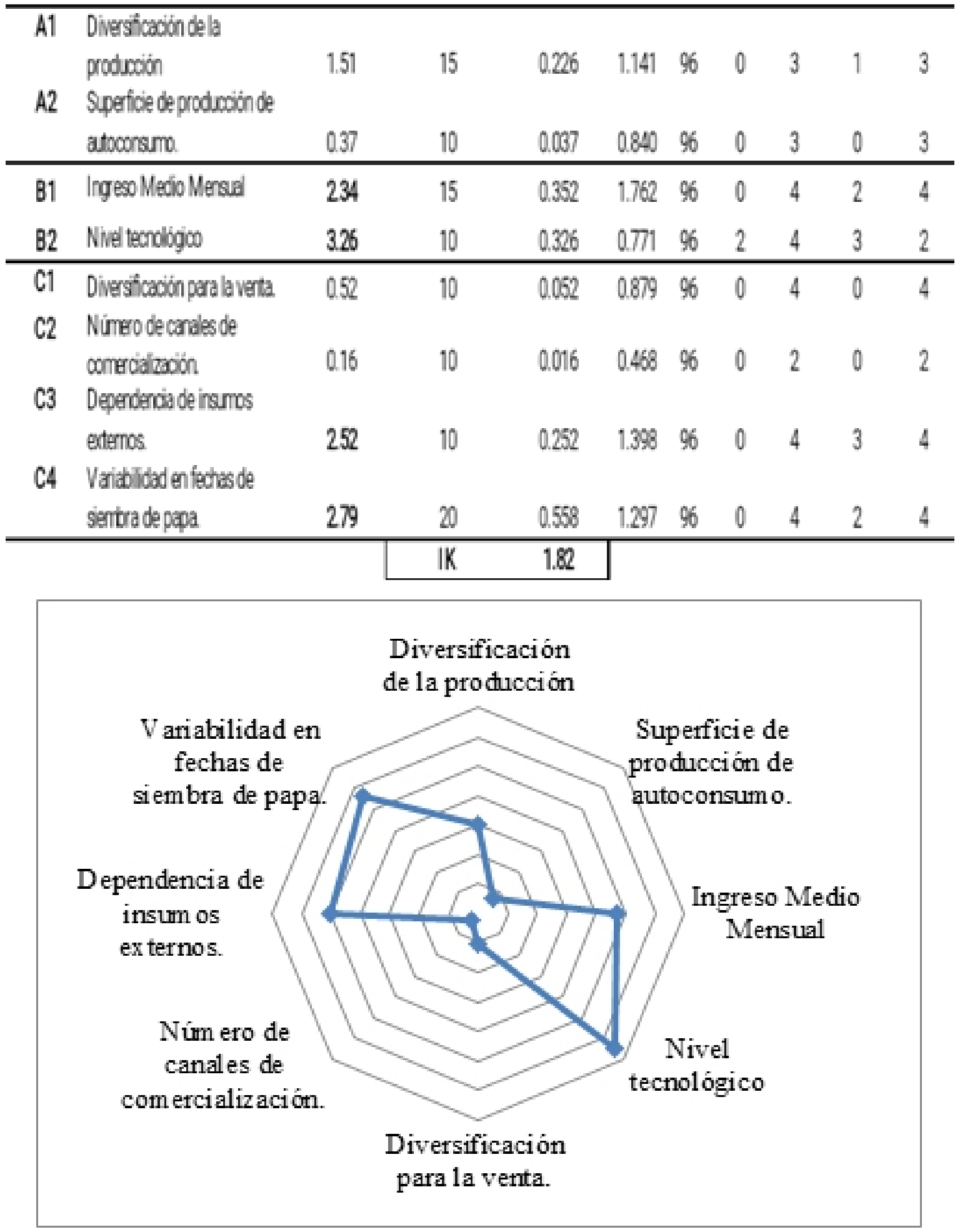

Figura 1. Indicadores de sustentabilidad económica de las fincas productoras de papa en la región Lima. 
Índice ponderado de sustentabilidad económica $=[0,15(1.51)+0,10(0.37)]+$ $[0,10(2.34)+0,10(3.26)]+[0,10(0.52)$ $+0,10(0.16)+0,10(2.52)+0,20(2.79)]=$

\section{$\mathbf{I E}=\mathbf{1 . 8 2}$}

\section{DISCUSIÓN}

En comparación al resto de cultivos no industriales, la papa representó en la región Lima el $14 \%$ de las intenciones de siembra durante la campaña 2014-2015 en la región, precedidos por el maíz amarillo duro (38\%) que ocupó el primer lugar (INEI, 2015).

De otro lado, la papa es un cultivo sensible a los estreses ambientales (FAO, 2008; Midmore, 1988) y algunos autores indican que las variables climáticas como las temperaturas máximas tienen efectos negativos en el rendimiento de los cultivos de papa (Tonconi, 2015).

La información obtenida de las fuentes oficiales del rendimiento de papa que alcanzó una media de $26.02 \mathrm{t} \mathrm{ha}^{-1}$, (M IN A GRI, 2015 ) coincide cercanamente con la obtenida de las encuestas realizadas en la presente investigación, ya que el valor medio obtenido a través de encuestas en la región Lima fue de $27.40 \mathrm{tha}^{-1}$.

De acuerdo con la información proporcionada por la Dirección Regional de Agricultura MINAGRI (2015), el costo de producción de papa en la región Lima ascendía a 18429 nuevos soles por hectárea de los cuales el $35.9 \%$ (6 615 nuevos soles) correspondía a costos indirectos. Los datos tomados de la encuesta realizada en la investigación se acercan a este valor, ya que se enc $\pm_{\text {ntran }}$ en el rango de 161364674 nuevos soles por hectárea, lo que confirmaría la información estadística.

El nivel tecnológico del productor de papa en la región puede considerarse de intermedio a alto tal como se puede apreciar en la tabla 10, tomando en cuenta que en la mayoría de los casos se ha podido establecer que utilizan regularmente seis o más de los factores esenciales que inciden en la productividad (mano de obra asalariada, mecanización, riego e insumos externos como fertilizantes y agroquímicos, entre otros inputs); este tipo de producción responde a las necesidades de materia prima exigidas por el mercado mayorista de Lima y los supermercados (Shimizu \& Scott, 2016).

Van der Zaag (2010) considera que la producción de papa ambientalmente sostenible requiere una menor dependencia de plaguicidas; los consumidores están ejerciendo cada vez más presión sobre la protección del medio ambiente y los agricultores están respondiendo con un uso reducido y más eficiente de los pesticidas; en la investigación se ha hallado que los productores de papa tienen una relativa dependencia por insumos externos y un medio a alto nivel tecnológico.

Los resultados muestran los valores estandarizados de los indicadores económicos propuestos según la metodología utilizada y finalmente el 
el valor estimado de dicho indicador ponderado (IE) que para el caso de las fincas productoras de papa en la región Lima fue de 1.82 equivalente a una débil sustentabilidad económica; el bajo valor hallado de la sustentabilidad económica en el estudio puede mostrar una amenaza futura para las fincas productoras de papa en Costa.

Una precaria autosuficiencia alimentaria y el mayor riesgo económico atribuido a los canales de comercialización así como la poca diversificación para la venta, fueron los indicadores de menor valor de sustentabilidad de los productores de papa en la región Lima. Los ingresos medios familiares, el nivel tecnológico, el nivel de dependencia de insumos externos y una oportuna época de siembra de papa fueron los indicadores que reportaron los más altos valores de sustentabilidad

\section{CONCLUSIONES}

De acuerdo con los resultados presentados se puede concluir que:

1) Entre los aspectos económicos más importantes que caracterizan el sistema de producción de papa en la región Lima, resaltan el rendimiento promedio que alcanza a 27.4 t ha-1, con un costo de producción de 16136 soles por hectárea. Los precios de venta en chacra fueron en promedio de 0.83 soles por kilo, lo que supone un valor bruto de la producción de 22742 soles y una utilidad media de 6606 soles por hectárea para la campaña 20142015, siendo el tamaño promedio del predio de papa en la región de 4.95 has.

2) Las fincas productoras de papa en la región Lima presentaron una débil sustentabilidad económica según la metodología propuesta, implicando que ello podría constituirse en una amenaza para la producción sostenible de papa en Costa.

1) Una precaria autosuficiencia alimentaria y el mayor riesgo económico atribuido a los canales de comercialización y la poca diversificación para la venta fueron los indicadores de menor valor de sustentabilidad de los productores de papa en la costa central.

2) Los ingresos medios familiares, el nivel tecnológico y una adecuada época de siembra de papa fueron los indicadores que reportaron los más altos valores de sustentabilidad.

\section{REFERENCIAS BIBLIOGRÁFICAS}

Altieri, M.A. 1997. Enfoque Agroecológico para el Desarrollo de Sistemas de Producción Sostenibles en los Andes. Ed. CIED. Lima-Perú. 92 p.

Banco Mundial. 2008. Informe sobre el desarrollo mundial. Agricultura para el desarrollo. Banco Mundial. Parte I, II y III.

CIP. 2014. Tackling Global Research D e v e lo p m e n t: C I P's Engagement Agenda . International Potato Center Annual Report 2013. Lima. 
June, 2014. 60 p.

Contreras, S.E., García, S. 2016. Caracterización Socioeconómica del Sistema de Producción de Papa en la Provincia de Barranca. Big Bang Faustiniano 5(2), 37 41.

Eguren, F. 2012. Eficiencia y rendimientos en la agricultura peruana. La revista Agraria No. 141, 11-13.

FAO. 2008. La economía mundial de la papa. El año internacional de la papa 2008. Secretaria del año internacional de la Papa. FAO. Roma

Instituto Nacional de Estadística e Informática (INEI). 2015. Compendio Estadístico Lima Provincias 2014. INEI. Lima. 588 p.

Maldonado, L; Suárez, V., Thiele. G. 2008. Estudio de la adopción de variedades de papa en zonas pobres del Perú. Centro Internacional de la Papa (CIP). Lima-Perú. Documento de Trabajo 2008-2.37 p.

Masera, O.; Astier, M. \& López-Ridaura, S. 1999. Sustentabilidad y manejo de recursos naturales. El Marco de Evaluación MESMIS. GIRA.A.C. México. 109 p.

Midmore, D. J. 1988. Fisiología de la planta de papa bajo condiciones de clima cálido. Centro International de la Papa. Guía de investigación CIP24.16 p.
M I N A G R I . 2013 . D i nám i c a Agropecuaria 2002-2013. Oficina de Estudios Económicos y Estadísticos. Ministerio de Agricultura y Riego. Lima.

MINAGRI. 2015. Intenciones de siembra 2015-2016. DGESEP. Ministerio de Agricultura y Riego. Lima. Julio 2015.

R Core Team. 2015. R: A language and environment for statistical computing. R Foundation for Statistical Computing, Vienna, Austria. ISBN 3-900051-07-0, URL http://www.R-project.org/

Sarandón, S. J., Flores, CC. 2014. Agroecología : bases teóricas para el diseño y manejo de agroecosistemas sustentables. 1a ed. La Plata: Universidad Nacional de La Plata. 467 P.

Sarandón, S. J. 1998. The development and use of sustainability indicators: a need for organic agriculture evaluation. XII International Scientific Conference IFOAM 1998. 16/19 Noviembre 1998, Mar del Plata, Argentina:pp. 135.

Sarandón, S.J. 2002. El desarrollo y uso de indicadores para evaluar la sustentabilidad de los agroecosistemas. En: Santiago J. Sarandón (editor): Agroecología. El camino hacia una Agricultura sustentable. Ediciones Científicas Americanas Capítulo 20:393-414. 
Scheaffer, R., Mendenhall, W. \& Ott, L. 1987. Elementos de muestreo. Editorial Iberoamericano. México DF.

Shimizu, T., Scott, G. 2016. Los supermercados y cambios en la cadena productiva para la papa en el Perú. Revista Latinoamericana de la Papa, 18(1), 77-103

Tonconi, J. 2015. Producción agrícola alimentaria y cambio climático: un análisis económico en el departamento de Puno, Perú. Idesia (Arica), 33(2), 119-136

Van der Zaag, P. 2010. Toward sustainable potato production: experience with alternative methods of pest and disease control on a commercial potato farm. American Journal of Potato Research, 87(5), 428-433.

\section{CORRESPONDENCIA}

Dr. Sergio Contreras Liza

scontreras@unjfsc.edu.pe 